\title{
Effect of biscuits fortified with different doses of vitamin $A$ on indices of vitamin A status, haemoglobin and physical growth levels of pre-school children in Chongqing
}

\author{
Xuan Zhang ${ }^{1} \uparrow$, Ke Chen ${ }^{2} \uparrow$, Ping Qu ${ }^{3}$, You-Xue Liu ${ }^{3}$ and Ting-Yu Li ${ }^{1,3, *}$ \\ ${ }^{1}$ Child Health Care, Children's Hospital, Chongqing Medical University, Chongqing 400014, People's Republic \\ of China: ${ }^{2}$ Chengdu Maternal and Children Health Care Hospital, 32 Shiye Street, Chengdu, Sichuan Province, \\ People's Republic of China: ${ }^{3}$ Children's Nutritional Research Center, Pediatric Research Institute, Chongqing \\ Medical University, Chongqing, People's Republic of China
}

Submitted 7 0ctober 2008: Accepted 2 March 2010: First published online 22 April 2010

\begin{abstract}
Objective: To investigate the efficacy of biscuits fortified with different doses of vitamin A on improving vitamin A deficiency (VAD), anaemia and physical growth of pre-school children.

Design: A randomised double-masked population-based field interventional trial with a positive control group.

Setting: Banan district of Chongqing, China.

Subjects: A total of 580 pre-school children aged 3-6 years were randomly recruited into four groups. Children in groups I and II were given biscuits fortified with vitamin $\mathrm{A}$ at $30 \%$ of the recommended daily intake (RDA) and $100 \%$ of the RDA once a day for 9 and 3 months, respectively. Children in group III received biscuits containing 20000 IU of vitamin A once a week for 3 months. Initially, the children in group IV received a $200000 \mathrm{IU}$ vitamin A capsule just once. At the beginning and end of the study, blood samples were collected to measure $\mathrm{Hb}$, serum retinol, retinol-binding protein and prealbumin, and weight and height were measured.

Results: All the fortification types significantly decreased the prevalence of VAD and anaemia in each group $(P<0 \cdot 05)$. The effect of 9 -month intervention on group I was the most efficient $(P<0 \cdot 0045)$. After intervention, the $Z$-scores of height-for-age, weight-for-age and weight-for-height in all groups increased markedly compared with baseline $(P<0 \cdot 05)$, but no significant difference was observed among the groups. Conclusions: Data indicated that consuming vitamin A-fortified biscuits with daily $100 \%$ RDA for 3 months has the same effect on the improvement of VAD, anaemia and physical growth as did the weekly 20000 IU and single 200000 IU administration in pre-school children.
\end{abstract}

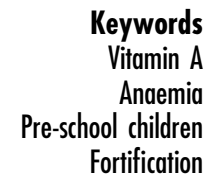

Fortification
Anaemia and vitamin A deficiency (VAD) are widespread public health problems associated with increased risk of morbidity and mortality, especially in young children ${ }^{(1)}$. The Chinese Food and Nutrition Surveillance System documented that the prevalence of anaemia in pre-school children remained at 16-20\% in the 15 years from 1990 to $2005^{(2)}$. Except for iron, other nutritional deficiencies, such as that of vitamin A, may also adversely affect haematopoiesis and lead to anaemia ${ }^{(3)}$. A national nutrition and health survey in $2002^{(4)}$ revealed an incidence of $45 \cdot 1 \%$ for marginal VAD and $9 \cdot 3 \%$ for VAD in Chinese children aged $3-12$ years. At present, VAD is more serious among pre-school children in China $^{(4)}$ because their diets mainly consist of rice with low iron availability and without preformed vitamin A or provitamin A.

$\dagger$ Both authors contributed equally to this work.
High-dose vitamin A supplements are recommended as part of the treatment ${ }^{(5)}$. However, clinical trials that examined the effect of a high dosage of vitamin A on children with diarrhoea or respiratory infections showed that it had either no efficacy ${ }^{(6)}$ or only minimum efficacy $^{(7)}$, or even adverse effects ${ }^{(8)}$. Small doses of vitamin A given regularly might be a better strategy to improve VAD status and anaemia and reduce morbidity but they have been rarely tested.

Recently, several randomised controlled trials ${ }^{(9,10)}$ have shown micronutrient-fortified biscuits to be a feasible, practical and effective way to improve the micronutrient status of primary-school children from the poor rural community. However, there is as yet no research to evaluate the effect of biscuits fortified with different doses of vitamin A on serum vitamin A nutritional indices, $\mathrm{Hb}$ and the physical growth levels of pre-school children. 
In the present study, we conducted a randomised controlled study to investigate the efficacy of biscuits fortified with different doses of vitamin A on improving serum vitamin $\mathrm{A}$ and $\mathrm{Hb}$ status, physical growth of preschool children aged 3-6 years in a suburb in Chongqing, China. We hypothesised that providing lower doses of vitamin A through daily provision of fortified biscuits was as efficacious in reducing the prevalence of VAD and anaemia as a higher dose of vitamin A administration.

\section{Subjects and methods}

\section{Subjects and etbical approval}

This intervention programme was performed in Banan district of Chongqing from March to December 2002. Approximately 1000 pre-school children aged 3-6 years were randomly recruited from about thirty kindergartens in the region. The eligibility criteria for participation were as follows: (i) apparently good health; (ii) Hb concentration $\geq 60 \mathrm{~g} / \mathrm{l}$; (iii) C-reaction protein (CRP) $<10 \mathrm{mg} / \mathrm{l}$; (iv) parental or guardian's approval for participating in all aspects of the study; and (v) parental/guardian's agreement to avoid additional use of vitamin and mineral supplements during the investigation. Children with evidence of recent acute or chronic illnesses and/or $\mathrm{Hb}$ $<60 \mathrm{~g} / \mathrm{l}$ were not included in the study and were referred to a local medical centre for treatment.

The primary outcome objective for the present study was the change between pre- and post-intervention in serum retinol concentration. A sample size of about 135 pre-school children per group was sufficient to allow the detection of a difference of $0.04 \mu \mathrm{mol} / 1$ in serum retinol concentration, $5 \cdot 3 \%$ prevalence of $\mathrm{VAD}$ and $13.0 \%$ prevalence of anaemia among the four interventions over 3 months with $90.0 \%$ power and $\alpha=0.05$ for two-sided one-way ANOVA. Considering the loss of children over the duration of the study, we initially managed to recruit more than 200 children per group. However, the primary outcome measures including analyses of micronutrient status of blood and anthropometric indices before and after intervention were obtained from 580 pre-school children, due to the loss to follow-up in the present trial.

Informed written consent was obtained from the parents/guardians. The enrolment and research plan were reviewed and approved by the institutional ethics committee of the Children's Hospital of Chongqing Medical University in Chongqing, China.

Information on the socio-economic and demographic parameters of the children and their families was collected.

\section{Intervention}

The present study used fortified biscuits directly for oral application with dairy products, such as bean milk or soup. The biscuits of all four packages were similar in macronutrient composition $(71.3 \mathrm{~g}$ carbohydrate, $8.0 \mathrm{~g}$ protein, $16 \cdot 0 \mathrm{~g}$ fat, $2 \cdot 2 \mathrm{~g}$ fibre and $1856 \cdot 8 \mathrm{~kJ}$ per $100 \mathrm{~g}$ biscuit) and were indistinguishable in taste, colour and packaging, except for the coded numerical labelling. Package I biscuits contained $30 \%$ of the recommended daily intake (RDA; set by the Chinese Nutrition Society) for vitamin A (500 IU as dry vitamin A acetate) per piece, and package II had $100 \%$ of the RDA for vitamin A (1666 IU as dry vitamin A acetate) per biscuit piece. Package III contained biscuits with supplements of 20000 IU of vitamin A (as dry vitamin A acetate) per piece, while package IV had no vitamin A and was used as placebo biscuit. All of the biscuits were provided by Shanghai Danone Biscuits Foods Co., Ltd (Shanghai, China) (2002-2003) and the concentration of fortification was determined by Shanghai Roche Pharmaceuticals Ltd (Shanghai, China).

The study was designed as a double-blinded, randomised and positive-controlled trial. With the help of child health-care workers, the children who met the eligibility criteria in all of the nurseries were randomly assigned to one of the four groups within the sex and age strata to help ensure sex and age balance of treatment allocation. The RAND function of Excel (Microsoft, Redmond, WA, USA) was used to generate computer randomly permutated codes. Pre-school children in groups I and II were given fortified biscuits packages I and II once a day for 9 and 3 months, respectively. Children in group III were provided with biscuit package III once a week for the interval time for 3 months. Lastly, children in group IV received a 200000 IU vitamin A capsule (as retinyl ester) just once initially and then biscuit package IV for the rest of the time for 3 months.

Fortified biscuits were given to each child at afternoon snack time, $5 \mathrm{~d}$ /week during the study period for all groups. Children in group III were given biscuit package III only on every Monday afternoon. No intervention took place during school holidays or public holidays, and the biscuits were provided for a total of 59 school days (the equivalent of 12 weeks). The investigators, food preparers, teachers, outcome assessors and children were not made aware of the intervention assignment for the duration of the study until after the completion of data analysis.

\section{Blood sample collection and biochemical measurement or assessment}

At the beginning and at the end of the 3-month period (children in group I who received two measurements or assessments at the end of the 3-month period were designated as group I-3, and those who received measurements at the end of the 9-month period were designated as group I-9), two or three (for children in group I) blood samples (about $1.5 \mathrm{ml}$ ) were collected by venepuncture of an antecubital vein from each subject before breakfast. A volume of $0.5 \mathrm{ml}$ was drawn into a container with heparin to measure $\mathrm{Hb}$ by the hemiglobincyanide 
method $^{(11)}$ (MAKER, China). The concentrations of serum retinol-binding protein (RBP) and prealbumin (PA) were measured by ELISA ${ }^{(12)}$ with commercial ELISA kits (Sunbiote, China). CRP was measured by particle-enhanced immunoturbidimetry ${ }^{(13)}$ (catalogue no. 67274; Orion Corporation Orion Diagnostica, Finland). Serum retinol concentration was determined by using HPLC following the method of Miller and Yang with slight modification ${ }^{(14)}$. All of the biochemical indices were measured by experienced examiners in the Pediatric Laboratory of Chongqing Medical University.

\section{Antbropometric measurements}

Anthropometric examinations in any one group were conducted by the same trained anthropometrist (a total of four) from the Chongqing Children's Hospital at the baseline and follow-up (3- or 9-month) time points using standardised techniques to eliminate intra-examiner error. By using reference data from the US National Center for Health Statistics/WHO (2005), Z-scores were calculated for height-for-age (HAZ), weight-for-height (WHZ) and weight-for-age (WAZ). A cut-off of less than minus two standard deviations $(-2 \mathrm{SD})$ was used to define stunting (HAZ $<-2$ ) and wasting (WHZ $<-2$ ), while a cut-off of more than plus two standard deviations $(+2 \mathrm{SD})$ was used to define obesity (WHZ $>+2)^{(15)}$. All indices were computed using the ANTHRO software (Anthro 2005 PC) recommended by WHO (http://www.who.int/childgrowth/ software/en/).

Simultaneously, assessment of symptoms and signs of VAD (xerophthalmia, Bitot's spots and/or night blindness) of pre-school children was performed by paediatric oculists.

\section{Definition of outcomes}

The prevalence of deficiency for some biochemical indices was expressed as the percentage of pre-school children below the cut-off value. Prevalence of anaemia was determined according to the WHO criteria, i.e. $\mathrm{Hb}<110$ $\mathrm{g} / \mathrm{l}$ for 5-month to 6-year-olds and $\mathrm{Hb}<115 \mathrm{~g} / \mathrm{l}$ for 6-11-year-olds ${ }^{(16)}$. According to the WHO criteria ${ }^{(17)}$, a serum retinol concentration of $<0.7 \mu \mathrm{mol} / 1$ was classified as VAD and values between 0.70 and $1.05 \mu \mathrm{mol} / 1$ were defined as marginal vitamin A deficiency (MVAD). CRP levels of $>10 \mathrm{mg} / \mathrm{l}$ indicated infection or inflammation as recommended by the manufacturer (Roche Diagnostics).

\section{Statistical analysis}

Using the Kolmogorov-Smirnov goodness-of-fit test, the distribution of each set of data was tested for normality prior to analysis. Data were presented as the mean and SD for normally distributed variables or median (25th, 75th) for skewed distribution variables and, when necessary, data were normalised using natural-log transformations. Tests of significance were two-tailed and $P<0.05$ was considered statistically significant. Paired Student's $t$ tests were used to compare paired data with normal distribution and homogeneous variance in the before-after intervention pairs of each group, while paired Wilcoxon sign-rank test was used for skewed distribution data. The $\chi^{2}$ test was used for categorical variables, with a Bonferroni correction for multiple comparisons among multiple groups. In order to compare the different effects among the four interventions, we performed multiple comparisons of the changes of parameters over the 3-month intervention $(P<0.05$ set for statistical significance) with Student-Newman-Keuls (SNK) test (normal and homogeneous data) or SNK rank test (skewed data). For SNK test or SNK rank test, the SNK grouping letters were provided. Data were analysed using the SAS for Windows statistical software package version $8 \cdot 1$ (SAS Institute Inc., Cary, NC, USA).

\section{Results}

Seven hundred and fifty-three children met the inclusion criteria (177 for group I, 173 for group II, 209 for group III and 194 for group IV). About 23.0\% (173/753) dropped out during the course of the study. The dropout number was forty-two (four for blood drawing failure and thirty-eight for moved during trial) for group I, thirty-two (three for blood drawing failure and twenty-nine for moved during trial) for group II, forty-four (two for blood drawing failure and fortytwo for moved during trial) for group III and 55 (six for blood drawing failure and forty-nine for moved during trial) for group IV, respectively. Thus, primary outcome measures including analyses of micronutrient status of blood and anthropometric indices at the end of intervention were obtained from 580 pre-school children (135, 141, 165 and 139 from groups I, II, III and IV, respectively; Fig. 1). Retrospective data documented daily by caregivers indicated about $95 \%$ compliance, suggesting that at least $95 \%$ of the pre-school children took the biscuits during the study period.

\section{Baseline characteristics}

The demographic characteristics of the children studied are given in Table 1. There was no significant difference in clinical characteristics among the treatment group children who completed the study. Similarly, no marked difference in the parameters mentioned above was found between the children who completed the study and those who dropped out $(P>0 \cdot 05)$.

\section{Effects of intervention on the prevalence of vitamin A deficiency and anaemia}

At baseline, about $7 \cdot 6 \%(57 / 753)$ of the children had VAD and $34 \cdot 8 \%(262 / 753)$ of them had MVAD. The prevalence of anaemia was $26.6 \%(200 / 753)$ before intervention and $2 \cdot 0 \%(15 / 753)$ of the children had both VAD and anaemia. No significant difference was observed for the prevalence of $\operatorname{VAD}\left(\chi^{2}=0.97, P=0.81\right)$ and anaemia $\left(\chi^{2}=4 \cdot 89, P=0 \cdot 18\right)$ at baseline among the treatment groups. After the 3-month intervention, the four ratios 


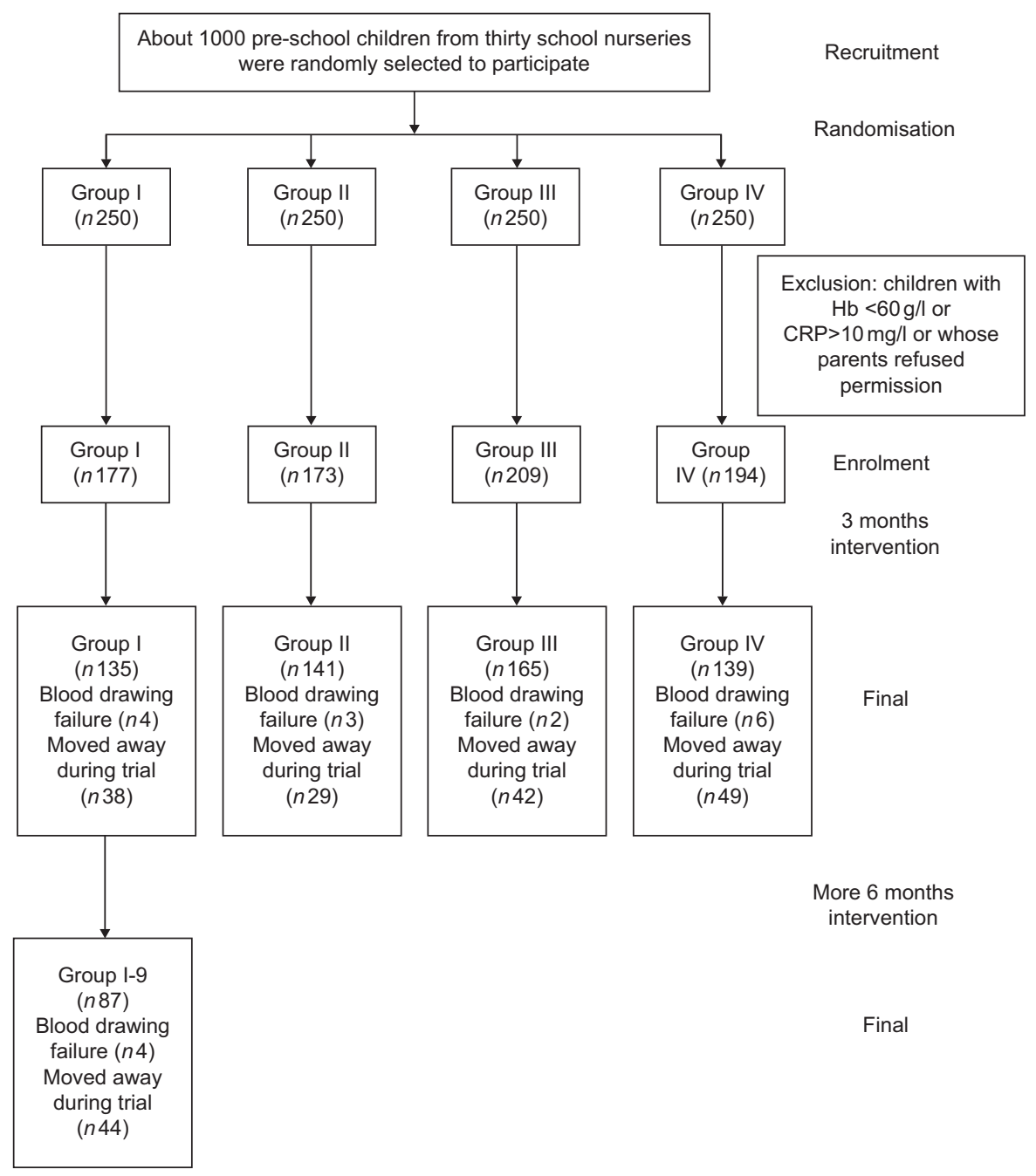

Fig. 1 Trial profile showing the number of participants randomly assigned, completing the trial, and analysis of the primary outcome (CRP, C-reactive protein)

mentioned above decreased to $1 \cdot 6 \%(9 / 580), 30 \cdot 5 \%$ (177/580), $10 \cdot 3 \%(60 / 580)$ and $0 \cdot 2 \%(1 / 580)$, respectively. Meanwhile, the prevalence of VAD and anaemia in all groups, including the children with the 9-month supplementation, decreased significantly $(P<0.05$; Table 2). No significant difference in the prevalence of VAD between groups after the intervention was found with the multiple comparative Fisher's exact test (Table probability $=0 \cdot 06, P=0 \cdot 36$ ). But the prevalence of anaemia after intervention in group I-9 was the lowest $(P<0 \cdot 0045)$ compared with the other four groups and no statistical difference in anaemia after intervention among the other four groups $(P>0.05)$ was observed with multiple comparative $\chi^{2}$ tests.

\section{Effects of intervention on serum biochemical indices and antbropometric measurements}

The concentrations of serum retinol, $\mathrm{Hb}, \mathrm{PA}$ and RBP and anthropometric measurement $Z$-scores, including HAZ, WAZ and WHZ, at baseline and at the end of the study, as well as the change over 3 or 9 months of intervention, are summarised in Tables 3 and 4 . At baseline, no significant difference in these indices was observed among the four groups $(P>0 \cdot 05)$. After the 3-month supplementation, the concentrations of serum retinol, RBP, HAZ, WAZ and WHZ in all groups increased markedly compared with the baseline $(P<0 \cdot 05)$, but those of PA and $\mathrm{Hb}$ increased only in groups II, III and IV. Meanwhile, the increase in serum retinol in children of group III was the highest compared with the other three groups, while that of group I was the lowest (both $P<0 \cdot 05)$. No statistical difference in the change of serum retinol was found between the group II and group IV children $(P>0 \cdot 05)$. Moreover, the increases in $\mathrm{Hb}, \mathrm{PA}$, HAZ and WHZ in group I were the lowest in comparison to the other three groups $(P<0 \cdot 05)$ and there was no significant difference in the changes in $\mathrm{Hb}, \mathrm{PA}, \mathrm{HAZ}$ and WHZ among groups II, III and IV $(P>0 \cdot 05)$. No significant difference in the changes in RBP and WAZ was observed among the four groups $(P>0 \cdot 05)$. 
Table 1 Demographic characteristics of treatment groups at baseline and comparison between the subjects who completed the study and who did not

\begin{tabular}{|c|c|c|c|c|c|c|c|c|c|c|c|c|}
\hline \multirow[b]{2}{*}{ Parameters } & \multicolumn{10}{|c|}{ Treatment group children who completed the study ${ }^{*}$} & \multirow{2}{*}{\multicolumn{2}{|c|}{ Dropped-out childrent }} \\
\hline & \multicolumn{2}{|c|}{ Group I } & \multicolumn{2}{|c|}{ Group II } & \multicolumn{2}{|c|}{ Group III } & \multicolumn{2}{|c|}{ Group IV } & \multicolumn{2}{|c|}{ Total } & & \\
\hline \multirow[t]{2}{*}{ Number of children } & \multicolumn{2}{|c|}{135} & \multicolumn{2}{|c|}{141} & \multicolumn{2}{|c|}{165} & \multicolumn{2}{|c|}{139} & \multicolumn{2}{|c|}{580} & \multicolumn{2}{|c|}{173} \\
\hline & Mean & SD & Mean & SD & Mean & SD & Mean & SD & Mean & SD & Mean & SD \\
\hline \multirow[t]{2}{*}{ Age (months) } & $50 \cdot 1$ & $7 \cdot 3$ & $\begin{array}{ll}49 \cdot 8 \\
4\end{array}$ & $9 \cdot 9$ & $50 \cdot 9$ & $8 \cdot 9$ & $51 \cdot 3$ & $10 \cdot 6$ & $50 \cdot 5$ & $9 \cdot 7$ & $50 \cdot 2$ & $9 \cdot 6$ \\
\hline & \multicolumn{2}{|c|}{$\%$} & \multicolumn{2}{|c|}{$\%$} & \multicolumn{2}{|c|}{$\%$} & \multicolumn{2}{|c|}{$\%$} & \multicolumn{2}{|c|}{$\%$} & \multicolumn{2}{|c|}{$\%$} \\
\hline Sex: male & \multicolumn{2}{|c|}{$50 \cdot 4$} & \multicolumn{2}{|c|}{$50 \cdot 4$} & \multicolumn{2}{|c|}{$50 \cdot 3$} & \multicolumn{2}{|c|}{$49 \cdot 6$} & \multicolumn{2}{|c|}{$50 \cdot 1$} & \multicolumn{2}{|c|}{$50 \cdot 3$} \\
\hline Diarrhoea in previous 2 weeks & \multicolumn{2}{|c|}{$4 \cdot 4$} & \multicolumn{2}{|c|}{$5 \cdot 7$} & \multicolumn{2}{|c|}{$4 \cdot 2$} & \multicolumn{2}{|c|}{$3 \cdot 6$} & \multicolumn{2}{|c|}{4.5} & \multicolumn{2}{|c|}{$4 \cdot 6$} \\
\hline Fever in previous 2 weeks & & & & & & & & & & & & \\
\hline Ever received vitamin A supplement & & & & & & & & & & & & \\
\hline Mother with high-school education & 40 & & & & & & & & & & & \\
\hline Father with high-school education & 51 & & & & & & & & & & & \\
\hline Ever breast-fed & 89 & & & & & & & & & & & \\
\hline Regular consumption $\ddagger$ of milk, liver and egg & 55 & & & & & & & & & & & \\
\hline Regular consumption $\ddagger$ of deep-coloured vegetables & 40 & & & & & & & & & & & \\
\hline $\mathrm{HAZ}<-2$ & & & & & & & & & & & & \\
\hline WHZ $<-2$ & & & & & & & & & & & & \\
\hline $\mathrm{WHZ}>+2 \mathrm{SD}$ & & & & & & & & & & & & \\
\hline
\end{tabular}

HAZ, height-for-age Z-score; WHZ, weight-for-height Z-score.

No xerophthalmia, Bitot's spots, and/or night blindness were observed.

${ }^{*}$ There was no significant difference in the parameters by treatment group in the children who completed the study, using the Student-Newman-Keuls test for the comparison of age, the $\chi^{2}$ test for the comparison of categorical variables with a Bonferroni correction and Fisher's exact test for the comparison of HAZ and WHZ.

tThere was no significant difference in the parameters between the total children who completed the study and the children who dropped out, using Student's $t$ test for the comparison of age, the $\chi^{2}$ test for the comparison of categorical variables and Fisher's exact test for the comparison of HAZ and WHZ.

†Regular consumption: consumption days of certain foods were more than or equal to $3 \mathrm{~d}$ in a week. 
Table 2 Comparison of prevalence of VAD and anaemia in treatment groups from baseline to 3-month follow-up for all groups and to 9-month for group I

\begin{tabular}{|c|c|c|c|c|c|c|c|c|c|c|}
\hline \multirow[b]{4}{*}{ Parameters } & \multicolumn{10}{|c|}{ Treatment groups } \\
\hline & \multicolumn{4}{|c|}{ Group I } & & & & & & \\
\hline & \multicolumn{2}{|c|}{ Group 1-3* } & \multicolumn{2}{|c|}{ Group 1-9* } & \multicolumn{2}{|c|}{ Group II } & \multicolumn{2}{|c|}{ Group III } & \multicolumn{2}{|c|}{ Group IV } \\
\hline & $\%$ & $n$ & $\%$ & $n$ & $\%$ & $n$ & $\%$ & $n$ & $\%$ & $n$ \\
\hline \multicolumn{11}{|l|}{ VAD } \\
\hline Baselinet & $6 \cdot 7$ & $12 / 177$ & $6 \cdot 5$ & $11 / 173$ & $8 \cdot 6$ & $18 / 209$ & $8 \cdot 3$ & $16 / 194$ & $6 \cdot 7$ & $12 / 177$ \\
\hline After interventionł & $1 \cdot 5$ & $2 / 135^{a}$ & $1 \cdot 4$ & $2 / 141^{a}$ & $1 \cdot 2$ & $2 / 165^{a}$ & $2 \cdot 2$ & $3 / 139^{a}$ & 0.0 & $0 / 87^{a}$ \\
\hline Values of $P \S$ & $<0.05$ II & & $<0.05 \|$ & & $<0.01 \|$ & & $<0.0511$ & & $<0.05 \|$ & \\
\hline \multicolumn{11}{|l|}{ Anaemia } \\
\hline Baselinet & $21 \cdot 5$ & $38 / 177$ & $30 \cdot 6$ & $53 / 173$ & $29 \cdot 2$ & $61 / 209$ & $24 \cdot 7$ & $48 / 194$ & $21 \cdot 5$ & $38 / 177$ \\
\hline After intervention $\ddagger$ & $12 \cdot 6$ & $17 / 135^{a}$ & $9 \cdot 9$ & $14 / 141^{a}$ & $7 \cdot 3$ & $12 / 165^{a}$ & $12 \cdot 2$ & $17 / 139^{a}$ & $1 \cdot 2$ & $1 / 87^{b}$ \\
\hline Values of $P \S$ & $<0.05$ & & $<0.019$ & & $<0.019$ & & $<0.019$ & & $<0.01 \|$ & \\
\hline
\end{tabular}

VAD, vitamin A deficiency.

${ }^{*}$ Group I-3 and group I-9, 3- and 9-month follow-up of group I, respectively.

+No significant difference in the prevalence of VAD or anaemia was found at baseline in the treatment groups with the $\chi^{2}$ test.

‡Comparison of the prevalence of VAD or anaemia between groups after intervention with the multiple comparative $\chi^{2}$ test for adjusted $\alpha^{\prime}=0 \cdot 0045$. Values within a row with unlike superscript letters $\left({ }^{\mathrm{a}, \mathrm{b}}\right)$ were significantly different.

§Comparison of the prevalence of VAD or anaemia between baseline and after invention in treatment groups.

$11 \chi^{2}$ test.

-Fisher's exact test.

Table 3 Comparison of biochemical indices in treatment groups from baseline to 3-month follow-up for all groups and to 9-month for group I*

\begin{tabular}{|c|c|c|c|c|c|c|c|c|c|c|}
\hline \multirow[b]{4}{*}{ Variables } & \multicolumn{10}{|c|}{ Treatment groups } \\
\hline & \multicolumn{4}{|c|}{ Group I } & & & & & & \\
\hline & \multicolumn{2}{|c|}{ Group I-3 (n 135)† } & \multicolumn{2}{|c|}{ Group I-9 (n 87)† } & \multicolumn{2}{|c|}{ Group II (n 144) } & \multicolumn{2}{|c|}{ Group III (n 165) } & \multicolumn{2}{|c|}{ Group IV (n 139) } \\
\hline & Mean & SD & Mean & SD & Mean & SD & Mean & SD & Mean & SD \\
\hline \multicolumn{11}{|c|}{ Serum retinol $(\mu \mathrm{mol} / \mathrm{l}) \ddagger$} \\
\hline Baseline & 1.09 & 0.29 & 1.02 & $0 \cdot 25$ & 1.05 & $0 \cdot 28$ & 1.06 & $0 \cdot 26$ & $1 \cdot 13$ & $0 \cdot 29$ \\
\hline After intervention & $1 \cdot 17$ & $0 \cdot 27$ & $1 \cdot 15$ & $0 \cdot 27$ & $1 \cdot 27$ & $0 \cdot 32$ & $1 \cdot 18$ & $0 \cdot 28$ & $1 \cdot 21$ & $0 \cdot 2$ \\
\hline Changes & $0.08 \|$ & 0.29 & $0 \cdot 13 \|$ & $0 \cdot 29$ & $0 \cdot 23 \|$ & 0.33 & $0 \cdot 12 \|$ & $0 \cdot 31$ & $0.08 \|$ & $0 \cdot 29$ \\
\hline $\begin{array}{l}\text { SNK of change§ } \\
\text { PA }(\mathrm{mg} / \mathrm{l}) \pm\end{array}$ & & C & & B & & A & & B & & C \\
\hline \multicolumn{11}{|l|}{$\mathrm{PA}(\mathrm{mg} / \mathrm{l}) \ddagger$} \\
\hline Baseline & $220 \cdot 68$ & $47 \cdot 79$ & $211 \cdot 39$ & $43 \cdot 51$ & $218 \cdot 81$ & $51 \cdot 65$ & $221 \cdot 79$ & $63 \cdot 46$ & $228 \cdot 65$ & $47 \cdot 99$ \\
\hline After intervention & $236 \cdot 25$ & $55 \cdot 85$ & $244 \cdot 79$ & $50 \cdot 28$ & $244 \cdot 60$ & $52 \cdot 30$ & $255 \cdot 42$ & $57 \cdot 54$ & $299 \cdot 55$ & $62 \cdot 65$ \\
\hline \multirow{3}{*}{$\begin{array}{l}\text { Changes } \\
\text { SNK of change }\end{array}$} & $15 \cdot 57 \|$ & $60 \cdot 52$ & $33 \cdot 3911$ & $59 \cdot 65$ & $25 \cdot 79 \|$ & $62 \cdot 77$ & $33 \cdot 63 \|$ & $76 \cdot 54$ & $70 \cdot 9011$ & $70 \cdot 55$ \\
\hline & & C & & B & & B & & \multirow{2}{*}{ Percentiles } & \multirow{2}{*}{\multicolumn{2}{|c|}{ Percentiles }} \\
\hline & & Percentiles & & Percentiles & & Percentiles & & & & \\
\hline RBP (mg/l)‡ & Median & 25th 75th & Median & 25th 75th & Median & 25th 75th & Median & 25th 75th & Median & 25th 75th \\
\hline Baseline & $30 \cdot 00$ & $9 \cdot 23 \quad \overline{45 \cdot 77}$ & $30 \cdot 54$ & $\overline{12 \cdot 09} \overline{52 \cdot 31}$ & $29 \cdot 03$ & $\overline{7 \cdot 65} \overline{49 \cdot 61}$ & $32 \cdot 22$ & $\overline{10 \cdot 39} \overline{58.03}$ & $31 \cdot 70$ & $\overline{13 \cdot 33} \overline{49 \cdot 09}$ \\
\hline After intervention & $33 \cdot 76$ & $11.3950 \cdot 21$ & $33 \cdot 40$ & $12 \cdot 5961 \cdot 19$ & $33 \cdot 05$ & $9.98 \quad 57.13$ & $37 \cdot 19$ & $15 \cdot 4469 \cdot 03$ & $33 \cdot 40$ & 14.5554 .01 \\
\hline $\begin{array}{l}\text { Changes } \\
\text { SNK of change§ }\end{array}$ & $3 \cdot 76 \|$ & $\begin{array}{ll}1 \cdot 01 & 8 \cdot 73 \\
A & \end{array}$ & $2 \cdot 86 \|$ & $\begin{array}{ll}0.73 & 5.66 \\
A & \end{array}$ & $4 \cdot 02 \|$ & $\begin{array}{ll}1.98 & 7 \cdot 92 \\
A & \end{array}$ & $4 \cdot 97 \|$ & $\begin{array}{ll}2 \cdot 11 & 10 \cdot 39 \\
\mathrm{~A} & \end{array}$ & $1 \cdot 69 \|$ & $\begin{array}{ll}0.29 & 2.04 \\
\text { B } & \end{array}$ \\
\hline $\mathrm{Hb}(\mathrm{g} / \mathrm{l}) \ddagger$ & Mean & SD & Mean & SD & Mean & SD & Mean & SD & Mean & SD \\
\hline Baseline & $114 \cdot 61$ & $6 \cdot 74$ & $113 \cdot 34$ & $10 \cdot 13$ & $114 \cdot 12$ & $8 \cdot 84$ & $114 \cdot 26$ & $7 \cdot 76$ & $114 \cdot 56$ & $7 \cdot 61$ \\
\hline After intervention & $115 \cdot 93$ & 6.04 & $120 \cdot 32$ & $8 \cdot 84$ & $119 \cdot 82$ & 8.03 & $119 \cdot 48$ & $9 \cdot 61$ & $127 \cdot 10$ & $8 \cdot 86$ \\
\hline $\begin{array}{l}\text { Changes } \\
\text { SNK of change } \$\end{array}$ & $1 \cdot 32$ & $\begin{array}{l}7 \cdot 68 \\
C\end{array}$ & $6 \cdot 98 \|$ & $\begin{array}{l}9 \cdot 83 \\
B\end{array}$ & $5 \cdot 70 \|$ & $\begin{array}{l}9 \cdot 06 \\
B\end{array}$ & $5 \cdot 23 \|$ & $\begin{array}{l}9 \cdot 86 \\
B\end{array}$ & $12 \cdot 54 \|$ & $\begin{array}{l}9 \cdot 13 \\
\mathrm{~A}\end{array}$ \\
\hline
\end{tabular}

PA, prealbumin; RBP, retinol binding protein; SNK, Student-Newman-Keuls test.

*Data are presented as mean and SD for normally distributed variables or median (25th, 75th percentiles) for skewed distribution variables.

tGroup I-3 and group I-9, 3- and 9-month follow-up of group I, respectively.

$\ddagger$ At baseline, no significant difference was observed among the four treatment groups $(P>0.05)$.

§NK rank test or SNK test for multiple comparisons; within a row, unlike letters $(A, B, C, D)$ indicate significant difference.

IIAfter 3- or 9-month supplementation, the concentrations increased markedly compared with baseline.

Blood and anthropometric measurements were obtained from only eighty-seven children in group I at the end of 9-month supplementation (group I-9) because of children dropping out. However, the baseline biochemical indices and anthropometric measurement $Z$-scores in the eightyseven children were not statistically different compared with the other groups at baseline $(P>0 \cdot 05)$. Our data showed that the concentrations of serum retinol, $\mathrm{PA}, \mathrm{RBP}, \mathrm{Hb}, \mathrm{HAZ}$, 
Table 4 Comparison of anthropometric indices in treatment groups from baseline to 3-month follow-up for all groups and to 9-month for group $\mathrm{I}^{*}$

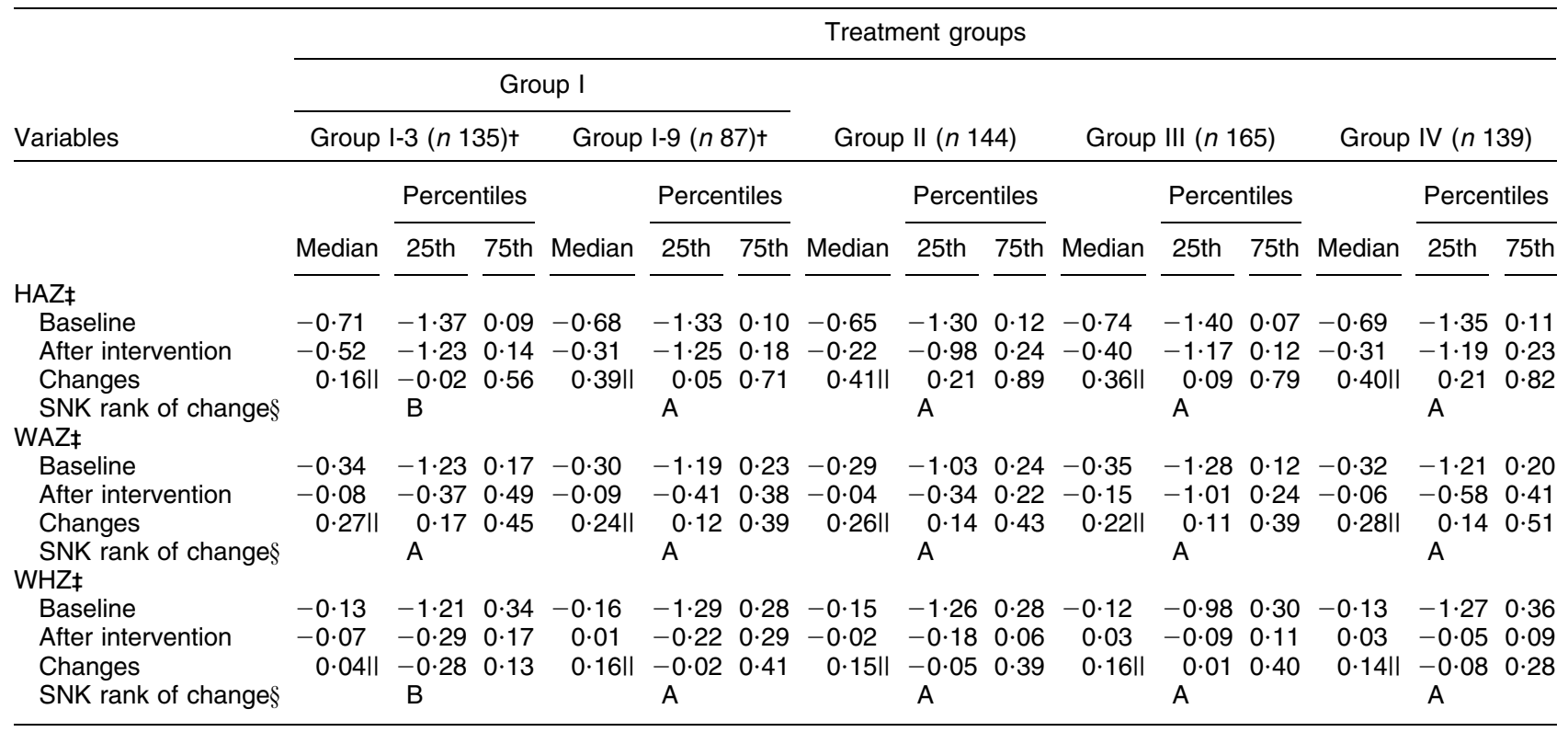

HAZ, height-for-age Z-score; WAZ, weight-for-age Z-score; WHZ, weight-for-height Z-score; SNK, Student-Newman-Keuls test.

${ }^{*}$ Data are presented as median (25th, 75th) as variables were non-normally distributed.

tGroup I-3 and group I-9, 3- and 9-month follow-up of group I, respectively.

$\ddagger$ At baseline, no significant difference was observed among the four treatment groups $(P>0.05)$.

$\S S N K$ rank test or SNK test for multiple comparisons; within a row, unlike letters $(A, B)$ indicate significant difference.

IIAfter 3- or 9-month supplementation, the levels increased markedly compared with baseline.

WAZ and WHZ increased significantly after 9-month supplementation $(P<0 \cdot 05)$. The changes in $\mathrm{PA}$ and $\mathrm{Hb}$ at the end of 9-month supplementation were the highest compared with the other four groups at the end of 3-month supplementation $(P<0 \cdot 05)$, but the changes in RBP were the lowest $(P<0 \cdot 05)$. The change in serum retinol was the same as that at the end of 3-month supplementation in group I (group I-3; $P>0 \cdot 05$ ). No significant changes in $\mathrm{HAZ}$ and WHZ at the end of 9-month supplementation were observed compared to groups II, III and IV $(P>0 \cdot 05)$, but they were higher than that in group I-3 $(P<0 \cdot 05)$. However, the levels in WAZ at the end of 9-month supplementation were similar to those of other groups $(P>0 \cdot 05)$.

\section{Discussion}

In the present study, we found that the intervention of biscuits fortified with vitamin A at $100 \%$ of the RDA daily for 3 months had the same effect on the improvement of VAD, anaemia and physical growth and development as did the weekly $20000 \mathrm{IU}$ or single $200000 \mathrm{IU}$ administration in preschool children in a suburb of Chongqing, China.

\section{Effects of intervention on the prevalence of $V A D$ and anaemia}

Ethical consideration precluded the use of an unsupplemented placebo group; therefore we just used a positivecontrolled strategy that enabled effective comparison of the new, different doses of a vitamin A supplementation schedule on vitamin A and anaemia status.

This randomised double-masked population-based field interventional trial with a positive control group showed that providing lower doses through fortified biscuits provided daily or weekly was as efficacious in reducing the prevalence of VAD and anaemia as highdose vitamin A administration. However, the present study did show the similar and greater efficacies of a daily low dose of vitamin A administration with extended duration in reducing the incidence of VAD and anaemia compared with the mass-dose vitamin A given. The major strength of the present study was the rigorous design and statistical analysis and the careful standardisation of the experimental measurement. To our knowledge, this is the first clinical trial to simultaneously exploit the effect of different doses of vitamin A intervention, including daily $30 \%$ RDA, daily $100 \%$ RDA, weekly 20000 IU and single $200000 \mathrm{IU}$, on the prevalence of VAD and anaemia in preschool children.

The WHO recommends that vitamin A supplements should be given when children are vaccinated, and the currently recommended doses are $100000 \mathrm{IU}$ at the age of 6-11 months and 200000 IU every 3-6 months at the age of $\geq 12$ months $^{(18)}$. There is no clear evidence, however, that a large dose is better than a smaller one. Serial population-based field trials ${ }^{(19-23)}$ have studied the effect of different administered doses of vitamin A on children's mortality and/or morbidity. Previous researches have 
implied that a low dose of vitamin A could always lower the mortality or the morbidity of children compared with a high dose and that the effect of supplementation might not be exclusively due to the prevention of vitamin A deficiency ${ }^{(24)}$. However, no integrated study has been performed to compare the effect of different doses of vitamin A supplements on the prevalence of VAD and anaemia in pre-school children, except for some researches about mass dose of vitamin A supplementation.

Recently, Darboe et al. ${ }^{(25)}$ pointed out that no difference in serum retinol level was found as one of the primary outcomes for high-dose vitamin A $v$. the WHO schedule, while the trial had shown possible adverse effects of higher doses of vitamin A consistent with results from Been and colleagues ${ }^{(23)}$. Another study ${ }^{(22)}$ also proved that a low dose of vitamin A (22500 IU) administered to infants is similar to substantially enhancing the biochemical vitamin A status of infants at the age of 6 months compared with a high dose of vitamin A supplement (45000 IU) and some vitamin A capsules degraded towards the end of the study. Moreover, Sivan et $a l^{(26)}$ documented that administration of $10 \mathrm{ml}$ red palm oil (including 800 retinol equivalent retinol) did not offer a substantial improvement in the serum retinol level over a $5 \mathrm{ml}$ daily dose (including 400 retinol equivalent retinol). One study ${ }^{(27)}$ reported that the intervention of a low dose of vitamin A did not improve the serum retinol status of children, but that was without any high-dose vitamin A intervention group for comparison. A similar research ${ }^{(28)}$ study also indicated that a low-dose vitamin A supplementation had no effect on the increase of $\mathrm{Hb}$ response. Although a field trial ${ }^{(29)}$ about vitamin A supplementation suggested that a high dose of vitamin A supplement could significantly improve serum retinol, mean $\mathrm{Hb}$ level and reduce the prevalence of anaemia compared with the control group, this trial lacked a lowdose vitamin A group as a control.

Our data indicated that the weekly administration of 20000 IU of vitamin A supplement was most efficacious on the change of concentration of serum retinol. However, this dosage was also the most cumulative dose (240 000 IU in total) compared with the other groups and a long-term use of such dose might increase the risk of vitamin A toxicity. Though daily 30\% RDA, 100\% RDA and a single dose of 200000 IU of vitamin A supplement had similar effects on the improvement of VAD, the former two supplement styles, based on the lower risk of toxicity of the RDA, might be the predominant doses for long-term intervention.

To our knowledge, there is no other study investigating the effect of different vitamin A supplement doses on vitamin A nutriture indicators, such as PA and RBP. In the present study, we found that the different doses of vitamin A had almost no effect on the concentrations of PA and RBP, suggesting that these indicators are buffered by the normal hepatic homoeostatic regulation of retinol stores and distribution, and hence are only effective at the extremes of vitamin A status. Nevertheless, they continue to be indicators of vitamin A status recommended by the WHO and the International Vitamin A Consultative Group $^{(12)}$, and help to confirm that the population we studied had mild vitamin A deficiency, as evidenced by about $7.57 \%$ prevalence of values below $0.7 \mu \mathrm{mol} / 1$ in pre-school children.

Remarkably, our findings were in partial agreement with some observations that single-dose supplements, repeated-dose supplements and high vitamin A intake do not alter retinol bioavailability; conversely, such intakes are associated with greatly increased exposure to toxic metabolites of vitamin A, such as retinyl palmitate, 13-cisretinoic acid and 13-cis-4-oxo-retinoic acid ${ }^{(30)}$. The wellknown phototoxicity and photomutagenicity of these compounds, which are linked to the generation of reactive oxygen species ${ }^{(31)}$, could be of concern and osseous side-effects have also been shown in children and adults treated with 13-cis-retinoic acid ${ }^{(32)}$.

Our data also indicated that the daily $30 \%$ RDA vitamin A with 9-month supplementation seemed to be more effective in decreasing the prevalence of anaemia and improving the $\mathrm{Hb}$ level. However, considering the various factors that might affect the results in the duration, such as the growth of the children with some possible changes in vitamin $\mathrm{A}$ and $\mathrm{Hb}$ status, diet and diseases, etc., we could not conclude whether 30\% RDA vitamin A with 9-month intervention style was the best. Further studies are required to compare the effects of different doses of vitamin A intervention for a long equal duration, to approach a more prominent way to improve anaemia.

\section{Effect of intervention on antbropometric measures}

The results showed that compared with pre-treatment, linear growth was similarly increased significantly for all of the preschool children in the five supplement groups. Therefore, fortified biscuits might not only prevent the deterioration of anthropometric status but also improve it. Comparisons did not show the additional effect of a mass dose of vitamin A on anthropometric measures when contrasted with weekly $20000 \mathrm{IU}$ and daily 100\% RDA supplements. However, contrary to our data, some other studies have suggested that mass-dose vitamin A had no effect on children's anthropometry, growth velocity, Z-scores, or body composition compared with the placebo-controlled group ${ }^{(33-35)}$.

Measurement of the dose that gives the maximum reduction in the prevalence of VAD and anaemia and/or the increase of physical growth is difficult and without the knowledge of the mechanisms by which the reduction in VAD and anaemia is achieved, many large-scale empirical trials are needed. Potential adverse effects of vitamin A supplementation are difficult to predict and surrogate predictors might assist in tailoring supplementation in different environments. 
There are some limitations in the current study. The effects of fortified biscuits on anthropometric status need to be investigated further and protective packaging of the biscuits for oral application must also be used. Furthermore, because of the limited design of the original protocol and the inadequate amount of blood samples obtained from these pre-school children, we did not simultaneously evaluate some other confounding factors, such as seasonal components and other essential nutrients (serum zinc, iron, or unsaturated fatty acid, etc.), which are capable of affecting anaemia and physical growth. Measuring these micronutrient levels will be an important aspect in future studies. In addition, regardless of the investigation of the regular consumption of milk, liver, egg and deep-coloured vegetables, we did not measure dietary intakes of nutrients by $24 \mathrm{~h}$ recall questionnaires, which might lead to insufficient evaluation of the effect of pre-school children's dietary habits on their nutritional and growth status.

In summary, our results indicate that consumption of vitamin A-fortified biscuits with daily 100\% RDA for 3 months has the same effect on the improvement of VAD, anaemia and physical growth and development as did the weekly 20000 IU or single 200000 IU administration in pre-school children in a suburb of Chongqing, China.

\section{Acknowledgements}

The present study was supported by grants from the SIGHT AND LIFE Research Institute, a humanitarian initiative of DSM, Municipal Education Commission, Chongqing, China, and grants from the National Natural Science Foundation of China (no. 30830106 and no. 30600495). The authors do not have any possible conflicts of interest. X. Zhang did the project design, data acquisition, analysis and interpretation, article draft and revision; K. Chen did the project design, data acquisition, analysis and interpretation, article draft and revision; P. Qu did the project design, data analysis and interpretation and technical assistance; Y-X. Liu did the project conception and design, data analysis and interpretation and general supervision; T-Y. Li did the project conception and design, data analysis and interpretation, article draft and revision and general supervision. The authors thank all the children and their parents for participating in the study. The authors also thank the health-care workers in Banan Health Center for Women and Children of Chongqing, China. The authors are grateful to Tian Weiming, Wang Wuli and Liu Guanjun for their diligent assistance. The authors are also grateful for the kind assistance of Shanghai Danone Biscuits Foods Co., Ltd (Shanghai, China) and Shanghai Roche Pharmaceuticals Ltd (Shanghai, China).

\section{References}

1. Guilbert JJ (2003) The world health report 2002 - reducing risks, promoting healthy life. Educ Health (Abingdon) 16, 230.
2. Chen CM, He W, Fu ZY et al. (2006) Analysis on the changes of nutritional situation in past 15 years (1990-2005) in China: the 15-year establishment of Chinese food and nutrition surveillance system (CFNSS). Wei Sheng Yan Jiu 35, 762-764, 774

3. Fishman SM, Christian P \& West KP (2000) The role of vitamins in the prevention and control of anaemia. Public Health Nutr 3, 125-150.

4. Li LM, Rao KQ, Kong LZ et al. (2005) A description on the Chinese national nutrition and health survey in 2002. Zhonghua Liu Xing Bing Xue Za Zhi 26, 478-484.

5. Ross DA (2002) Recommendations for vitamin A supplementation. J Nutr 132, Suppl. 9, 2902S-2906S.

6. Faruque AS, Mahalanabis D, Haque SS et al. (1999) Doubleblind, randomized, controlled trial of zinc or vitamin A supplementation in young children with acute diarrhoea. Acta Paediatr 88, 154-160.

7. Julien MR, Gomes A, Varandas L et al. (1999) A randomised, double-blind, placebo-controlled clinical trial of vitamin A in Mozambican children hospitalised with non-measles acute lower respiratory tract infections. Trop Med Int Health 4, 794-800.

8. Mahalanabis D, Lahiri M, Paul D et al. (2004) Randomized, double-blind, placebo-controlled clinical trial of the efficacy of treatment with zinc or vitamin A in infants and young children with severe acute lower respiratory infection. Am J Clin Nutr 79, 430-436.

9. van Stuijvenberg ME, Kvalsvig JD, Faber M et al. (1999) Effect of iron-, iodine-, and beta-carotene-fortified biscuits on the micronutrient status of primary school children: a randomized controlled trial. Am J Clin Nutr 69, 497-503.

10. van Stuijvenberg ME, Dhansay MA, Lombard CJ et al. (2001) The effect of a biscuit with red palm oil as a source of beta-carotene on the vitamin A status of primary school children: a comparison with beta-carotene from a synthetic source in a randomised controlled trial. Eur J Clin Nutr $\mathbf{5 5}$, 657-662.

11. International Committee for Standardization in Haematology; Expert Panel on Haemoglobinometry (1987) Recommendations for reference method for haemoglobinometry in human blood (ICSH standard 1986) and specifications for international haemiglobincyanide reference preparation (3rd edition). Clin Lab Haematol 9, 73-79.

12. Tanumihardjo SA (2004) Assessing vitamin A status: past, present and future. J Nutr 134, 290S-293S.

13. Eda S, Kaufmann J, Roos W et al. (1998) Development of a new microparticle-enhanced turbidimetric assay for C-reactive protein with superior features in analytical sensitivity and dynamic range. J Clin Lab Anal 12, 137-144.

14. Miller KW \& Yang CS (1985) An isocratic high-performance liquid chromatography method for the simultaneous analysis of plasma retinol, alpha-tocopherol, and various carotenoids. Anal Biochem 145, 21-26.

15. Kuczmarski RJ, Ogden CL, Grummer-Strawn LM et al. (2000) CDC growth charts: United States. Adv Data issue $314,1-27$.

16. World Health Organization/UNICEF/United Nations University (1998) Iron Deficiency: Indicators for Assessment and Strategies for Prevention. Geneva: WHO.

17. World Health Organization (1994) Indicators for Assessing vitamin A Deficiency and Their Application in Monitoring and Evaluating Intervention Programmes. Report of a WHO/UNICEF Consultation, Geneva, 9-11 November 1992. Geneva: WHO.

18. World Health Organization/UNICEF/International Vitamin A Consultative Group Task Force (1997) Vitamin A Supplements: A Guide to Their Use in the Treatment and Prevention of Vitamin A Deficiency and Xerophthalmia. Geneva: WHO. 
19. Donnen P, Dramaix M, Brasseur D et al. (1998) Randomized placebo-controlled clinical trial of the effect of a single high dose or daily low doses of vitamin A on the morbidity of hospitalized, malnourished children. Am J Clin Nutr 68, 1254-1260.

20. Semba RD, Munasir Z, Akib A et al. (2001) Integration of vitamin A supplementation with the expanded programme on immunization: lack of impact on morbidity or infant growth. Acta Paediatr 90, 1107-1111.

21. Donnen P, Sylla A, Dramaix M et al. (2007) Effect of daily low dose of vitamin A compared with single high dose on morbidity and mortality of hospitalized mainly malnourished children in senegal: a randomized controlled clinical trial. Eur J Clin Nutr 61, 1393-1399.

22. Idindili B, Masanja H, Urassa $\mathrm{H}$ et al. (2007) Randomized controlled safety and efficacy trial of 2 vitamin A supplementation schedules in Tanzanian infants. Am J Clin Nutr 85, 1312-1319.

23. Benn CS, Martins C, Rodrigues A et al. (2005) Randomised study of effect of different doses of vitamin A on childhood morbidity and mortality. BMJ 331, 1428-1432.

24. Benn CS, Bale C, Sommerfelt H et al. (2003) Hypothesis: vitamin A supplementation and childhood mortality: amplification of the non-specific effects of vaccines? Int $J$ Epidemiol 32, 822-828.

25. Darboe MK, Thurnham DI, Morgan G et al. (2007) Effectiveness of an early supplementation scheme of high-dose vitamin A versus standard WHO protocol in Gambian mothers and infants: a randomised controlled trial. Lancet 369, 2088-2096.

26. Sivan YS, Alwin Jayakumar Y, Arumughan C et al. (2002) Impact of vitamin A supplementation through different dosages of red palm oil and retinol palmitate on preschool children. J Trop Pediatr 48, 24-28.

27. Sazawal S, Dhingra U, Deb S et al. (2007) Effect of zinc added to multi-vitamin supplementation containing low-dose vitamin A on plasma retinol level in children - a double-blind randomized, controlled trial. J Health Popul Nutr 25, 62-66.

28. Alarcon K, Kolsteren PW, Prada AM et al. (2004) Effects of separate delivery of zinc or zinc and vitamin A on hemoglobin response, growth, and diarrhea in young Peruvian children receiving iron therapy for anemia. $\mathrm{Am} \mathrm{J}$ Clin Nutr 80, 1276-1282.

29. Zimmermann MB, Biebinger R, Rohner $\mathrm{F}$ et al. (2006) Vitamin A supplementation in children with poor vitamin A and iron status increases erythropoietin and hemoglobin concentrations without changing total body iron. Am J Clin Nutr 84, 580-586.

30. Hartmann S, Brors O, Bock J et al. (2005) Exposure to retinoic acids in non-pregnant women following high vitamin A intake with a liver meal. Int J Vitam Nutr Res 75, 187-194.

31. Yan J, Xia Q, Cherng SH et al. (2005) Photo-induced DNA damage and photocytotoxicity of retinyl palmitate and its photodecomposition products. Toxicol Ind Health 21, $167-175$.

32. DiGiovanna JJ (2001) Isotretinoin effects on bone. J Am Acad Dermatol 45, S176-SS82.

33. Sarni RS, Kochi C, Ramalho RA et al. (2003) Impact of vitamin A megadose supplementation on the anthropometry of children and adolescents with non-hormonal statural deficit: a double-blind and randomized clinical study. Int J Vitam Nutr Res 73, 303-311.

34. Mahawithanage ST, Kannangara KK, Wickremasinghe R et al. (2007) Impact of vitamin A supplementation on health status and absenteeism of school children in Sri Lanka. Asia Pac J Clin Nutr 16, 94-102.

35. Rahman MM, Tofail F, Wahed MA et al. (2002) Short-term supplementation with zinc and vitamin A has no significant effect on the growth of undernourished Bangladeshi children. Am J Clin Nutr 75, 87-91. 